\title{
Effect of different preparation heights and luting agents on tensile bond strengths in Nickel Chromium crown - a comparative in- vitro study
}

\author{
Dr. Sonali Babar ${ }^{1}$, Dr. S. V. Bhide ${ }^{2}$ Dr. Avantika jadhav ${ }^{3}$ \\ ${ }^{1 .}$ M.D.S. Department of Prosthodontics and Crown and Bridge, Bharati Vidyapeeth Deemed University, Dental \\ College and Hospital, Pune. \\ 2. H.O.D. Department of Prosthodontics and Crown and Bridge, Bharati Vidyapeeth Deemed University, \\ Dental College and Hospital, Pune. \\ ${ }^{3 .}$ M.D.S. Department of Prosthodontics and Crown and Bridge, Bharati Vidyapeeth Deemed University, Dental \\ College and Hospital, Pune.
}

\begin{abstract}
:
Aim: Mechanical characteristics of tooth preparation along with luting agents are significant entities on the maintenance of fixed dental prostheses. This study aimed at assessing the retention of complete metal crowns luted with two different luting agents using two different preparation height.

Methods: Forty human maxillary premolars were selected and prepared to receive complete metal crowns, and were randomly divided in 4 groups: Group A: 7-mm preparation height (PH) and glass ionomer cement (GIC); Group B: 7-mm PH and RelyX U200 self-adhesive resin cement (SA); Group C: 4-mm PH and glass ionomer cement (GIC); and Group D: 4-mm PH and RelyX U200 self-adhesive resin cement (SA). Crowns were casted in nickel-chromium alloy. The tensile strength was tested in a universal testing machine.

Results: Mean tensile strength for group Group A: $519.91 \pm 155.06$; for Group B: $643.81 \pm 142.65$; for Group C: $260.35 \pm 76.11$ and Group D: $627.95 \pm 209.34$.Overall, the crowns cemented with RelyX U200 self-adhesive resin cement presented significantly higher mean tensile strength values than GIC, and the 7-mm PH presented significantly higher mean tensile strength values than 4-mm PH.
\end{abstract}

Conclusions: The self-adhesive resin cement and $7 \mathrm{~mm}$ preparation height improved crown retention.

Keywords: crown retention, luting agents, preparation heigh..

\section{Introduction}

Fixed prosthodontic treatment can offer exceptional satisfaction for both patient and dentist. It can transform an unhealthy, unattractive dentition with poor function into a comfortable, healthy occlusion greatly enhancing aesthetics. Fixed prosthodontics is capable of giving years of further service and maintaining optimal oral health. Fixed prostheses includes restoration or replacement of part of tooth or multiple missing teeth. The complete crown is the most frequently used restoration for repairing severely damaged teeth and as a retainer for a fixed partial denture. ${ }^{1}$ To achieve that success, however, requires meticulous attention to every detail from initial patient interview through active treatment phases to a planned schedule of follow-up care. The present invitro study involves use of 2 different tooth preparation heights and 2 different cements as luting agents. In routine clinical practice there are patients with reduced clinical crown height. In such cases, gaining good retention and resistance form for the tooth with less height luted with conventional glass ionomer cement becomes difficult. ${ }^{2}$ Although there are few studies present on the relationship between tooth preparation height and retention of crown, in such patients, resin modified luting agent can be used which has good retentive and resistance properties such as RelyX U200 to increase crown retention. Therefore, the present study includes relationship between tooth preparation height and retention of metal crown. This in-vitro study involves fabrication of $\mathrm{Ni}-\mathrm{Cr}$ crown on extracted maxillary premolars with different preparation heights such as $7 \mathrm{~mm}$ and $4 \mathrm{~mm}$, which will be luted with conventional glass ionomer cement and RelyX U200.

\section{Material And Methods}

Forty human maxillary premolar teeth freshly extracted for orthodontic purpose were collected from Department of Oral and Maxillofacial Surgery, Bharati Vidyapeeth Deemed University, Dental College and Hospital, Pune. The extracted teeth were stored in normal saline during the course of study. The forty extracted maxillary premolars were prepared to receive a complete metal crown. The methodology was divided into 4 steps:-

1.1) Tooth preparation: A 14 gauge stainless steel rod was inserted transversely through the root of each premolars to ensure that tooth will not come out of the acrylic block during testing ( Fig 1). 40 wax blocks of 
dimension $20 \mathrm{~mm}$ length, $20 \mathrm{~mm}$ width and $20 \mathrm{~mm}$ height were made with modelling wax. Autopolymerizing resin was mixed in porcelain jar and poured in modelling wax block. While mounting the premolars in the acrylic resin block, they were placed above cemento-enamel junction. A surveyor was used to achieve parallelism between the long axis of the tooth and acrylic block ( Fig 2). The tooth preparation was done with the standard preparation features for full metal crown with adequate retention and resistance form ( Fig 3 ). The tooth preparation had following features: $:^{3,4}$

i) GROUP A and GROUP B: a) Occlusocervical dimension of $7 \mathrm{~mm}$, b) A $1 \mathrm{~mm}$ wide chamfer finish line, c) A 6-degree total convergence angle ( Fig 4).

ii) GROUP C AND GROUP D: a) Occlusocervical dimension of 4mm, b) A $1 \mathrm{~mm}$ wide chamfer finish line, c) A 6-degree total convergence angle ( Fig 5).

The standardization of tooth preparation was achieved by using an attachment of airotor to a surveyor. This attachment device consisted of a clamp, which was able to secure a high-speed airotor in a constant relationship with the surveyor. Its vertical relationship could be changed by the adjustable vertical spindle of the surveyor.

1.2) Casting: The wax patterns were directly fabricated on the prepared tooth ( Fig 6). After wax pattern fabrication was completed, a circular ring was made on each wax pattern for future testing it on the testing machine, using modelling wax. On the top flag was made to give sample number with the help of inlay wax and modelling wax. After the wax patterns were fabricated, investment of these wax patterns in phosphate bonded investment material ( Bellasum, Bego) was done. The investment material was then allowed to set for 45 minutes. Burnout was done. Five pellets of $\mathrm{Ni}-\mathrm{Cr}$ alloy were placed in the silica crucible and positioned properly in the casting machine. The alloy pellets were melted in the crucible through Electric Induction Furnace. The castings were then inspected for any nodules, and if present, were removed with a sintered diamond bur. Any casting with the defect was discarded and new casting was done. A total of 40 castings were fabricated in this manner ( Fig 7).

\section{3) Cementation: ${ }^{5,6,7}$ ( Fig 8)}

a) For GROUP A and GROUP C:- All 10 crowns were cemented with the help of conventional glass ionomer cement. Crown is seated on the tooth and is held under Compression load test setup under 5000gm for 5 minutes. b) For GROUP B and GROUP D:- All 10 crowns were cemented with the help of RelyX U2OO. crown is seated on the tooth and is held under Compression load test setup under 5000gm for 3 minutes.

1.4) Testing: After samples were cemented they were kept in water bath for 24 hours at 37 degree celcious. All samples were tested under Universal testing machine. Crown retention was measured by applying a tensile force to the circular ring attached to the cast crown in a universal testing machine at a cross-head speed of 0.5 $\mathrm{mm} / \mathrm{min}$. Each specimen was positioned on the lower part of the machine. The upper part of the testing machine had a steel hook which engages circular ring. Tensile force values required to separate the crowns (N) was recorded by machine's software. Statistical analysis was performed by one-way ANOVA. Descriptive statistics were expressed as mean \pm standard deviation (SD) for each group. Between and within group differences in tensile bond strength was analyzed using one way ANOVA test of significance with Bonferroni correction.

\section{Photos}

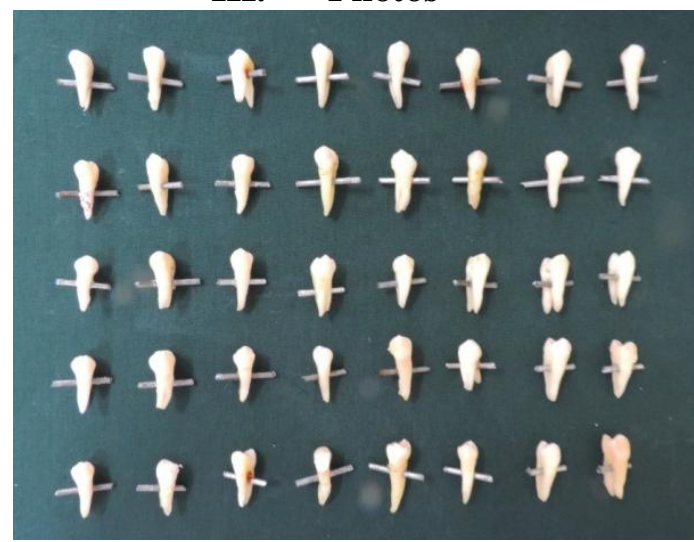

Fig. 1 Stainless steel rod inserted through extracted teeth. 


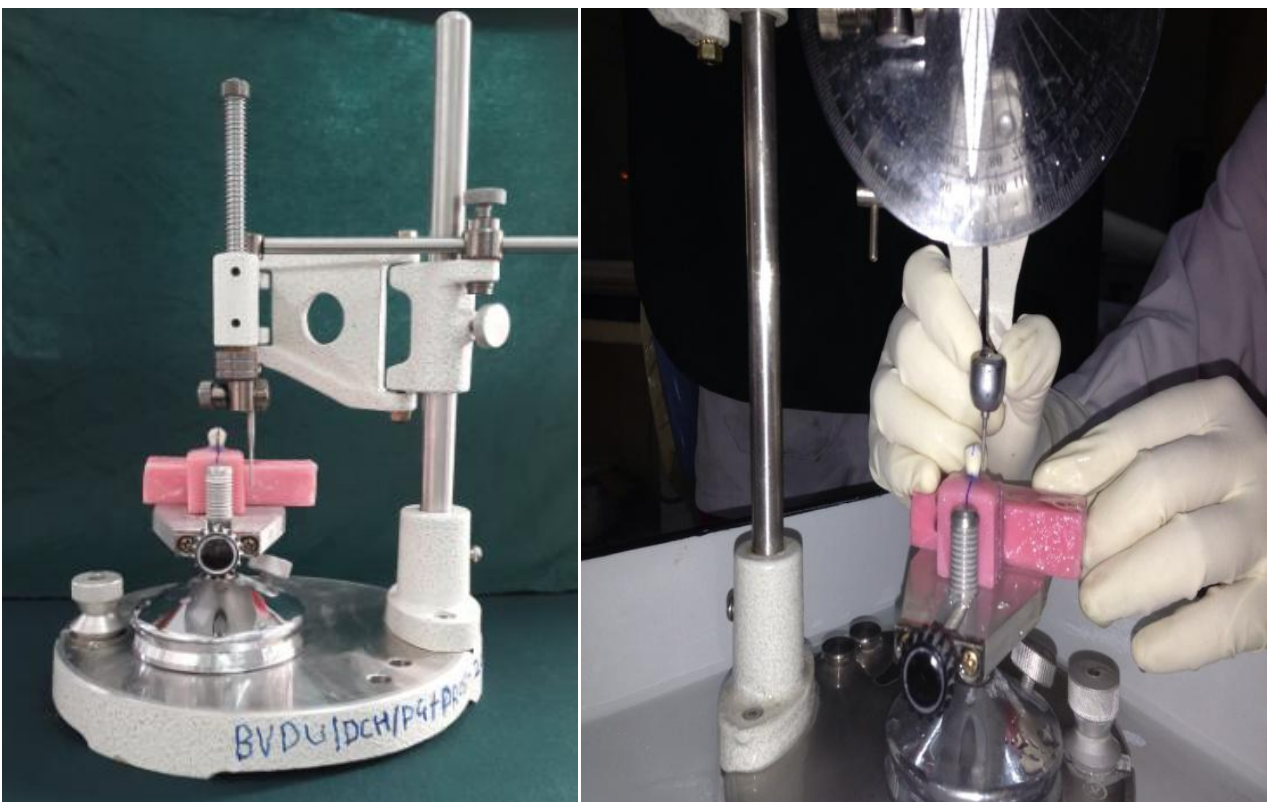

Fig 2: Surveyor used to acheive parallelism.

Fig 3: Tooth preparation

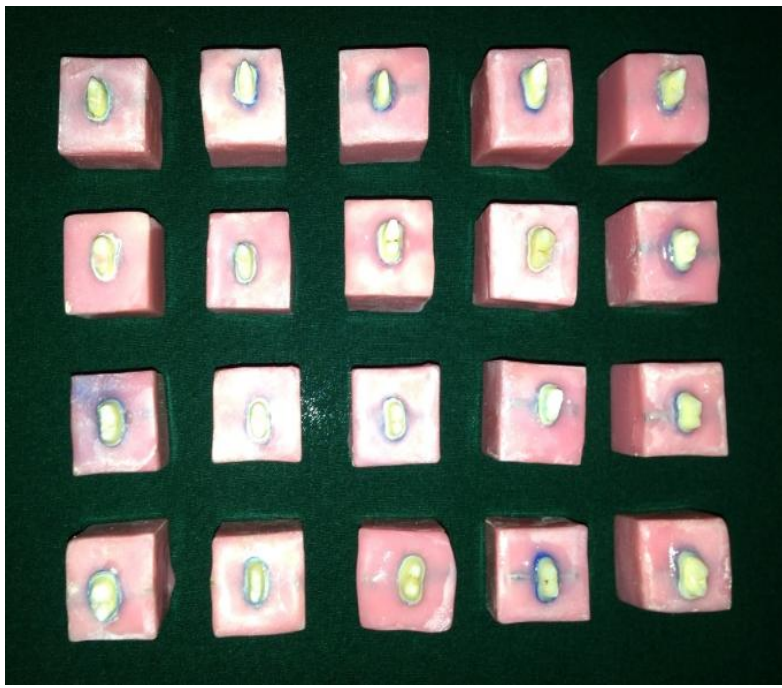

Fig 4: Group A and Group B

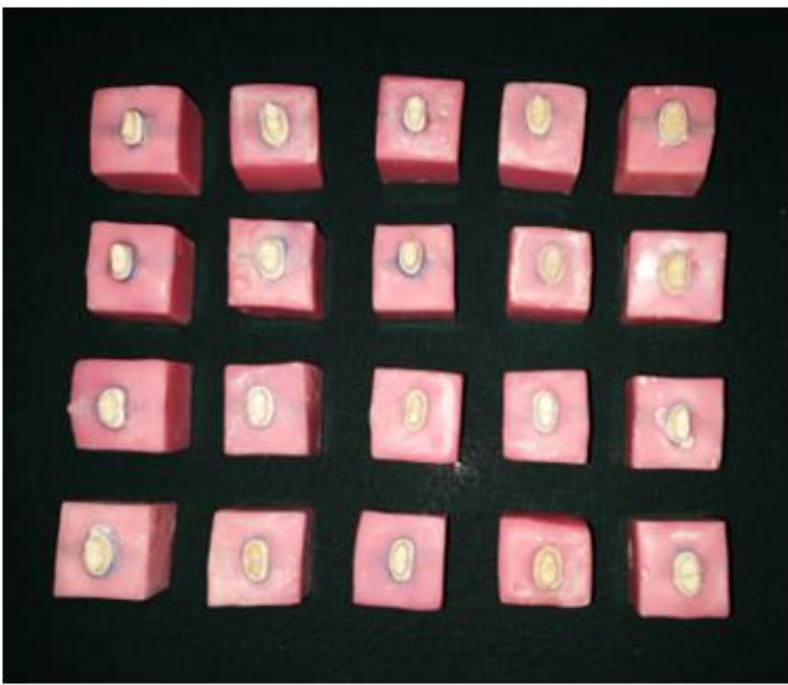

Fig 5: Group C and Group D 


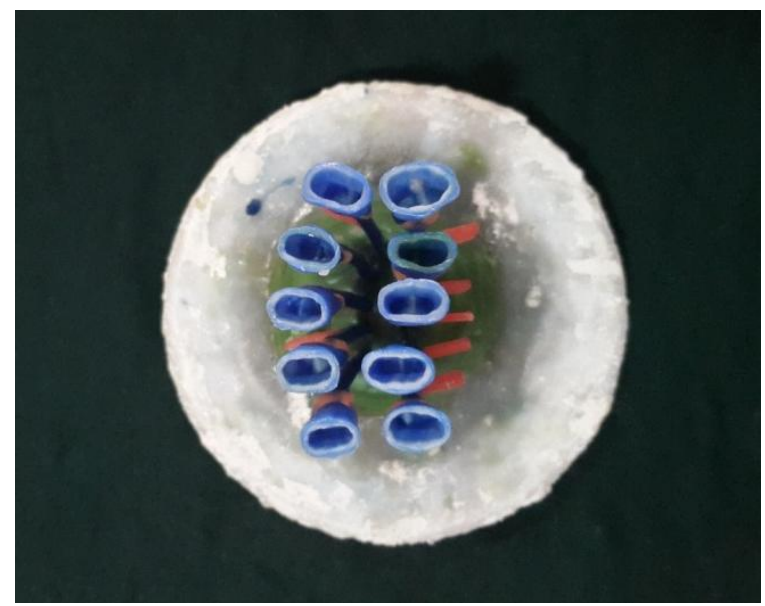

Fig 6: Wax patterns

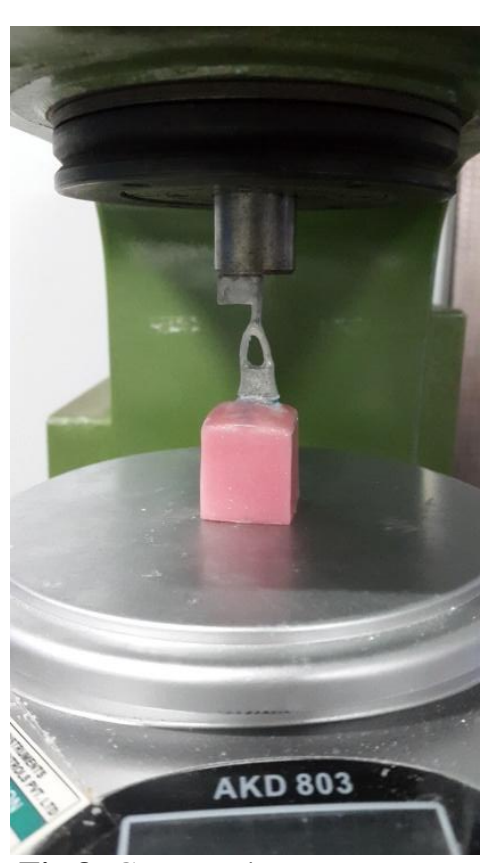

Fig 8: Cementation

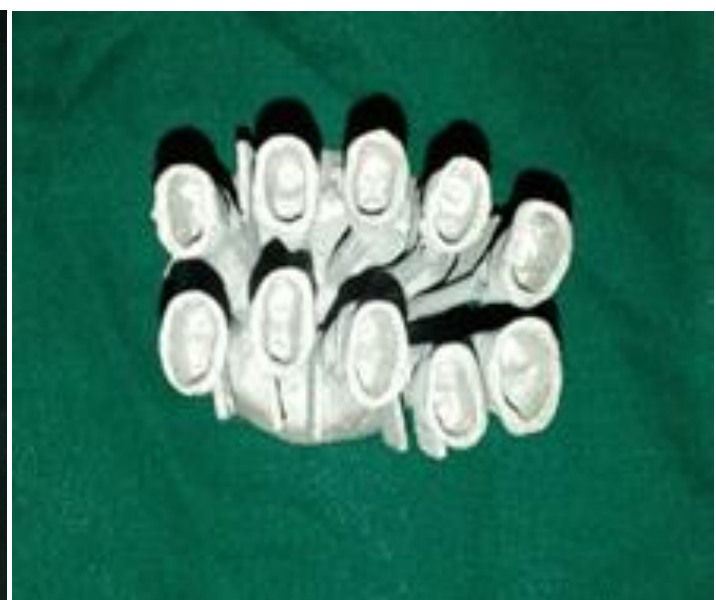

Fig 7: Castings

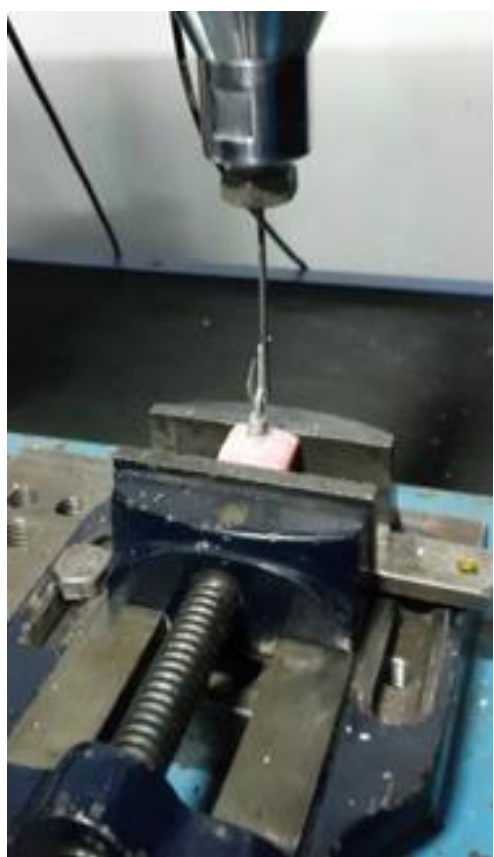

Fig 9: Testing

\section{Results}

All 40 metal crowns were tested for tensile strength with the help of Universal testing machine. Descriptive statistics were expressed as mean \pm standard deviation (SD) for each group. Between and within group differences in tensile bond strength was analyzed using one way ANOVA test of significance with Bonferroni correction. In the above test, $\mathrm{p}$ value less than or equal to 0.05 ( $\mathrm{p} \leq 0.05)$ was taken to be statistically significant. All analyses were performed using SPSS software version 10.

i) Mean tensile bond strength of Group A is $519.91 \pm 155.06$.

ii) Mean tensile bond strength of Group B is $643.81 \pm 142.62$.

iii) Mean tensile bond strength of Group C is $260.35 \pm 76.11$.

iv) Mean tensile bond strength of Group D is $627.95 \pm 209.34$.

Table 1: Comparison of effect of various preparation heights and luting agents on tensile strengths in $\mathrm{Ni}$-Cr

\begin{tabular}{|c|c|c|c|c|c|}
\hline \multicolumn{6}{|c|}{ crowns: } \\
\hline & Group A & Group B & Group C & Group D & P Value (ANOVA) \\
\hline $\begin{array}{l}\text { Tensile } \\
\text { strength } \\
(\text { mean } \pm \mathrm{SD})\end{array}$ & $\begin{array}{l}519.91 \\
\pm \\
155.06\end{array}$ & $\begin{array}{l}643.81 \\
\pm \\
142.62\end{array}$ & $\begin{array}{l}260.35 \\
\pm \\
76.11\end{array}$ & $\begin{array}{l}627.95 \\
\pm \\
209.34\end{array}$ & $<0.001 *$ \\
\hline
\end{tabular}


Post hoc Bonferroni test:

Mean tensile bond strength of,

Group B samples $>$ Group D samples, $\mathrm{p}=1.000$

Group D samples > Group A samples, $\mathrm{p}=0.625$

Group A samples $>$ Group C samples, $\mathrm{p}=\mathbf{0 . 0 0 2}$ *

${ }^{*} \mathrm{p} \leq 0.05$ is statistically significant.

i) When Group B was compared with Group D there was no statistically

significant difference seen.

ii) When Group D was compared with Group A there was no statistically

significant difference seen.

iii) When Group A was compared with Group C there was a statistically significant difference seen.

\section{Discussion}

Retention is considered an important requirement in the fixation of prosthetic crowns, and its achievement is dependent on some factors, namely favorable relation between surface geometry of the prepared tooth and the definitive restoration. Clinically, a crown would hardly undergo such great tensile efforts as those applied in this study, but the tested experimental conditions serve as parameters to evaluate different properties and behaviors of the materials used. ${ }^{8,9}$ The results of the present study may be explained by the fact that higher preparation height promotes greater superficial area with the crown. Although other factors may influence on crown retention, the preparations were standardized (cervical diameter, taper, roughness, piece fit), thus eliminating or minimizing the interference of these variables on the results. Concerning of the luting agents, the results showed greater retention for the RelyX U200 self- adhesive resin cement when compared to the glass ionomer cement, due to the adhesive capacity of the resin cement to dentin by hybrid layer formation, improving the retention compared to conventional cements. ${ }^{11}$ Moreover, better mechanical properties of resin cement in relation to glass ionomer cement also influence their tensile, compressive and shearing strengths. ${ }^{10}$ According to the obtained results, minimum occlusal reduction during preparation contributes significantly to increase the retention regardless of the type of luting agent used. The lower tensile strength of the GIC may be related to its ceramic composition, which makes this material friable and less resistant to tensile forces. Therefore, in situations where preparation retention is deficient, for example, a short clinical crown and accentuated taper of the preparation, the choice for a luting agent lies on resin cement, leading to a more favorable clinical prognosis. In cases of short clinical crown preparations where retention may be less for reasons mentioned above, retention can be increased by minimal TOC and use of an adhesive luting agents such as resin modified glass ionomer cements. ${ }^{12}$

The limitations of present study is that the tests do not simulate the exact clinical conditions therefore the results of exact clinical situations can differ from results of present study. It is important to note that the forces used to evaluate tensile bond strength in this study were considerably higher than the maximal physiologic forces acting on teeth intraorally.

\section{References}

[1]. Shillingburg HT, Hobo S, Whitsett LD, Jacobi R, Brackett SE. Fundamentals of fixed prosthodontics. 3rd ed. Chicago: Quintessence; 1997. p.139-42,151-2.

[2]. Potts RG, Shillingburg HT Jr, Duncanson MG Jr. Retention and resistance of preparations for cast restorations. J Prosthet Dent. 1980; 43: 303-8.

[3]. Leong EW, Choon Tan KB, Nicholls JI, Chua EK, Wong KM, Neo JC. The effect of preparation height and luting agent on the resistance form of cemented cast crowns under load fatigue. J Prosthet Dent. 2009; 102: 155-64.

[4]. Ayad MF, Johnston WM, Rosenstiel SF. Influence of tooth preparation taper and cement type on recementation strength of complete metal crowns. J Prosthet Dent. 2009; 102: 354-61.

[5]. Browning WD, Nelson SK, Cibirka R, Myers ML. Comparasion of luting cements for minimally retentive crown preparations. Quintessence Int.2002; 33: 95-100.

[6]. Piwowarczyk A, Lauer HC, Sorensen JA. In vitro shear bond strength of cementing agents to fixed prosthodontic restorative materials. J Prosthet Dent. 2004; 92: 363-76.

[7]. Radovic I, Monticelli F, Goracci C, Vulicevic ZR, Ferrari M. Self-adhesive resin cements: a literature review. J Adhes Dent. 2008; 10: $251-8$

[8]. Bowlwy JF, Kieser J. Axial-wall inclination angle and vertical height interactions in molar full crown preparations. J Dent. 2007; 35: 117-23.

[9]. Abbo B, Razzoog ME, Vivas J, Sierraalta M. Resistance to dislodgment of zirconia copings cemented onto titanium abutments of different heights. J Prosthet Dent. 2008; 99: 25-9.

[10]. Diaz-Arnold AM, Vargas MA, Haselton DR. Current status of luting agents for fixed prosthodontics. J Prosthet Dent. 1999; 81: $135-41$.

[11]. Tjan AHL, Li T. Seating and retention of complete crowns with a new adhesive resin cement. J Prosthet Dent. 1992; 67: 478-83.

[12]. Marcelo Marchiori. Influence of preparation height and luting agent type on crown retention in molars. Braz J Oral Sci, Vol. 9, No. 2, April-June, 2010, pp. 89-93. 